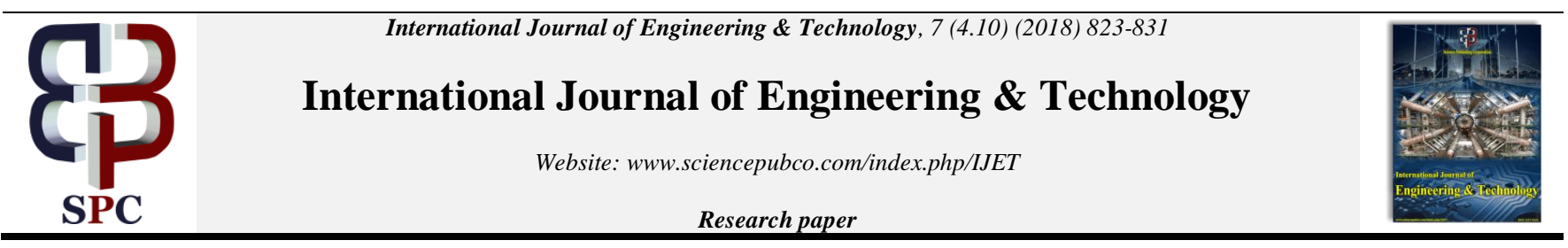

\title{
Road Extraction using Connected Component Techniques
}

\author{
J. D. Dorathi Jayaseeli ${ }^{1 *}$, D. Malathi ${ }^{2}$, Sarvesh Karandikar ${ }^{3}$ Aditi Singh $^{4}$ \\ Gopika $\mathbf{S}^{5}$ \\ ${ }^{1}$ Assitant Professor(S.G), CSE Department, SRM Institute of Science and Technology, Kattankulathur \\ ${ }^{2}$ Professor, CSE Department, SRM Institute of Science and Technology, Kattankulathur \\ ${ }^{3,4}$ B. tech Student CSE Department, SRM Institute of Science and Technology, Kattankulathur \\ ${ }^{5}$ Research Scholar, CSE Department, SRM Institute of Science and Technology, Kattankulathur \\ *Corresponding author E-mail: malathi.d@ktr.srmuniv.ac.in
}

\begin{abstract}
Extraction of Roads, Rivers and other map objects is an important step in many military and civilian applications. In this process the information is extracted which possess high efficiency and accuracy and is fed into GIS (Geographical Information System). In this paper, we have explored different algorithms with better efficiency and accuracy. Road extraction can take place for two kinds of roads namely: urban and non-urban roads. Urban roads are more complex to analyze because of their architectural complexity, occlusions created by trees, heavy traffic and extensive network, whereas non-urban roads are easier to analyze because of less structural complexity. The proposed algorithm exploits the properties of road segments to develop customized operators to accurately derive the road segments. The customized operators include directional morphological enhancement, directional segmentation and thinning. The proposed algorithm is systematically evaluated on the basis of variety of images and compared with other algorithms (Canny, Sobel, Roberts, and Morphological Segmentation). The results demonstrate that the algorithm proposed is both accurate and efficient. The data and performance measures such as completeness and correctness are calculated together with other parameters which are Peak Signal to Noise ratio, Normalized Cross Correlation, Structural Content and a statistical analysis of the comparison is presented.
\end{abstract}

Keywords: Connected Component, Adaptive Global,Thresholding, Morphological Operations, Normalized Cross Correlation, Structural Content

\section{Introduction}

Road extraction from satellite/aerial images is an area of image processing which is vastly researched to provide accurate and efficient experimental results. The area of road extraction is vast and many algorithms have been developed to provide effective results but none of the algorithm works effectively on all the images. Each and every algorithm has some or the other constraints which are yet to be overcome. The amount of research done in this field shows its importance in military, civilian and other fields which require prior knowledge of roads and lanes. The algorithms can be classified into semi-automatic, automatic and manual [1,2]. Manual method is easy to implement but requires lot of time while semi-automatic and automatic methods are harder to implement but can give effective and accurate results in less amount of time. While performing road extraction we focus on different structural and spectral features of the roads. The process of road detection generally consists of classification, segmentation and extraction. In order to discern the perfect extraction algorithm it is necessary that the algorithm focuses on different aspects of roads and different structural and spectral specificity's of roads.

Roads can be classified base on many different criteria and features. some of which involves the color, texture, geometric features, functional features and photo-metric features. On he basis of development roads can be classified as developed urban, developed sub-urban, developing urban and developing sub-urban. Developed urban can be defined as roads which are built in fully developed areas and hence are harder to detect because of the complexity involved in their structure. Developed sub-urban are the roads which are developed in less populated but fully developed areas. These roads are comparatively less difficult to extract than developed urban roads because of less. [3,4]

The history of road extraction can be linked to the earlier year of 70s. When remote sensing and its applications came into use the need of road extraction was felt, since then researchers started working in the area of image processing [5]. Firstly, road extraction was done manually wherein a person was appointed to highlight and draw roads and its features manually but as the process was very cumbersome the need for automation was felt hence came semi-automatic and automatic techniques. In semi -automatic technique image processing, segmentation, classification, optimization, edge detection, morphological segmentation came into light[6,7]. After the era of semi-automatic road extraction came the era of automatic road detection techniques which involved learning algorithms and artificial intelligence [8]. In automated road detection neural networks were used to give effective and efficient results with least human interference. These developments in the field of road extraction have increased the efficiency and accuracy of the targeted output but because of its vast application there can still be huge developments and improvements[9].

\section{Road detection algorithms-Overview}

\subsection{Sobel Edge Detection}

Sobel edge detection like other edge detectors operators are used to find edges of the given image and is used in computer vision or 
image processing algorithms[10]. Sobel operator is also a derivative mask and is very similar to Prewitt operators. It can be used to detect edges in two direction vertical and horizontal. In Sobel operators the mask used is different from other operators because its coefficients are not fixed that means we can adjust them as required keeping in mind the derivative operator. The vertical mask of Sobel operator is:

$\left[\begin{array}{lll}-1 & 0 & 1 \\ -2 & 0 & 2 \\ -1 & 0 & 1\end{array}\right]$

When we use this mask on the vertical edges it works as first order derivative and then calculated the difference in the intensities of the pixels in the direction of the edge. The zero in the centre column indicated that it does not uses the original values of the input image but rather it calculates difference between right pixel and left pixel[11]. The centre values which are 2 and -2 contain more weight and hence increase the intensity of edge detection and enhance the original image. The horizontal mask of Sobel operator is:

$\left[\begin{array}{ccc}-1 & -2 & -1 \\ 0 & 0 & 0 \\ 1 & 2 & 1\end{array}\right]$

This mask works on the horizontal edges of the given image. It also works similar to vertical mask in which it finds intensities between the two pixels of the particular edge in consideration. Here also the centre row is zero so it does not considers the value of the original image but rather it finds the difference between the above and below pixels.

\subsection{Roberts Edge Detection}

Roberts edge detection also works in a similar manner as other edge detector operator in which it finds the edge of the given image. It also makes use of a kernel or mask to perform its operations. It works by computing the image and hence finding its spatial gradient. It then highlights the regions having high spatial gradient hence giving the position of the edges[12]. The input of the operator is a gray scale image and the output is also a grayscale image. There are two masks for Roberts edge detection and they are:

$G x=\left[\begin{array}{cc}+1 & 0 \\ 0 & -1\end{array}\right], G y=\left[\begin{array}{cc}0 & +1 \\ -1 & 0\end{array}\right]$

\subsection{Canny Edge Detection}

Canny edge detection is a multi-step process which is used to detect edges in the image given as input. It takes input in the form of gray-scale image and hence detect intensities and discontinuities. These operators can be applied separately on the images to give separate values or can be applied together to give an absolute value[21].

In the multi stage process the first step is to smooth the image and it is done using Gaussian Convolution. Then a $2 \mathrm{D}$ operator or mask similar to the Roberts edge detection is applied to the image to highlight the regions of the image having high derivatives. In the gradient magnitude edges give rise to ridges. The algorithm uses this and tracks the tops of the ridges to give thin and accurate edges. It performs this by making all the pixels which are not present at the top of the ridge as zero. We can also use thresholds to get more effective outputs. operations. It works by computing the image and hence finding its spatial gradient. It then highlights the regions having high spatial gradient hence giving the position of the edges[13]. The input of the operator is a gray scale image and the output is also a gray-scale image. There are two masks for Roberts edge detection and they are:
$G x=\left[\begin{array}{cc}+1 & 0 \\ 0 & -1\end{array}\right], G y=\left[\begin{array}{cc}0 & +1 \\ -1 & 0\end{array}\right]$

These operators can be applied separately on the images to give separate values or can be applied together to give an absolute value.

\subsection{Canny Edge Detection}

Canny edge detection is a multi-step process which is used to detect edges in the image given as input. It takes input in the form of gray-scale image and hence detect intensities and discontinuities. In the multi stage process the first step is to smooth the image and it is done using Gaussian Convolution. Then a 2D operator or mask similar to the Roberts edge detection is applied to the image to highlight the regions of the image having high derivatives. In the gradient magnitude edges give rise to ridges. The algorithm uses this and tracks the tops of the ridges to give thin and accurate edges. It performs this by making all the pixels which are not present at the top of the ridge as zero. We can also use thresholds to get more effective outputs[15].

\subsection{K-means Segmentation}

Morphological Segmentation can be performed by following these steps: ridge finding, heuristic reasoning, dynamic programming, statistical inference, and map matching. Roads generally appears as ridges when we find the gray value function of the given image. Ridge finding uses edge detectors to give the magnitude of the edges and their directions which is followed by thinning and thresholding which gives us the pixels on the ridges. Heuristic reasoning uses knowledge which are there in the form of pre-set rules defining the characteristics of the roads and giving details about the road segments[16]. Dynamic Programming helps in modelling road pixels with the mathematical equations of their grayscale derivative characteristics of the roads and hence use them to perform optimization. Statistical inference gives details about the linear features and gives details about the road width, direction, background and directionalintensity

\section{Design and Analysis of Connected Compo- nent Method}

\subsection{Description}

The method consists of multi-step approach in which the main focus is on efficiency and accuracy. The steps involved are: Adaptive Global Thresholding, Connected Component Analysis, Morphological Closing, Morphological Opening, Morphological Thinning and Performance measures. The following modules when operated on the given data-set give high accuracy and efficiency. The dataset has been divided into the following 4 categories: Developing Urban, Developing Sub-Urban, Emerging Urban, Emerging Sub-Urban[17].

\subsection{Preprocessing}

This module helps in noise reductions. We have removed unnecessary noise using Gaussian Filter. The image is blurred horizontally and vertically using a one-dimensional kernel. The same onedimensional kernel is used to blur the image in the remaining directions. By following this step it is increasing the visual effect of the image. This in-turn will help in our geographical interpretations. This step will further help in improving the contrast between the desired output image and the input image[18]. A good function will help us in reducing the brightness of the darker areas on the roads but will not affect any non-targeted areas. The techniques 
used here is morphological operators which are: Dilation and Erosion.

\subsection{Adaptive Global Thresholding}

In this module the input image is converted into gray-level image and then the regions containing roads are extracted from it by making use of the process named as Histogram Analysis. This step is applied because it helps in removing non-road pixels and segments the regions containing roads from the given images. The process is carried forward by analyzing the histogram of the given image which is then divided into four main sections to obtain our desired threshold values which can be used for segmentation. The histogram of the given image can be analyzed based on the mean values of all the given pixels intensities of the following image. First section gives details about the areas containing dark objects which can be vehicles, shadows, lakes, ponds etc. Second section gives details about the about the region from half of Mean value to the Mean value and classifies dark gray objects such as trees, grasslands etc. Third Section gives details about the bright gray areas such as roads, lanes, parking area etc. Fourth, and the last section is used or identifying bright objects like cement road, bright vehicles etc[19]. The desired pixels are assigns with value as 1 and all the other pixels are given the value as 0 . Lastly, we convert the gray image in the binary image so that our road appears as white and all the other regions having non-road pixels appear as black.

\subsection{Connected Component Analysis}

Connected Component analysis for any given pixel can be defined as any set of pixels that is connected to a given pixel is called as connected component of the given image. Pixels which are not separated with the help of boundaries are called connected pixels. Connected component is the maximal region of each pixel. Connected Components help in partitioning the given image into segments. Connected component analysis is generally used for automatic image processing applications such as road extraction, object extraction, lines and lanes detection. Morphological operators can be used to extract connected components. We can apply morphological dilation and intersection operation for the extraction of connected components. The labeling under connected component is performed by moving pixel to pixel which can be from top to bottom or from left to right. This will help us in determining regions of adjacent pixels sharing same intensities. After performing scanning and labeling simultaneously the equivalent labels are searched and then each class is assigned with an unique label. In the last step again a scan is matched replacing each label with the label assigned to its equivalence classes.

\subsection{Morphological Closing}

Morphological function is an important operator in our system of modules. Its similar to its dual operator which is opening because it is derived using operations of erosion and dilation. It is applied to the binary images and can also be applied to some gray- level images. Its similar to dilation because it helps in the enlargement of boundaries of the bright regions which are also known as foreground regions of the images. It also shrinks the darker regions also known as background and hence helps in filling holes. It does not destructs the original boundary of the image very much. Structuring elements are used to perform the same operations in other morphological operators. The operator helps in preserving the background regions that are similar looking like the structural element or which can contain the structural element fully while eliminating all the other regions present in the background pixels. We can say that closing is opening performed in the reverse manner. It can be achieved by performing dilation followed by erosion and by making use of the same structural elements for performing both the operations. The closing operator requires 2 inputs which are: an image which we desire to close and an structuring element that is effective. One of the uses of dilation is to fill small holes which are similar in background color. The example of this type of hole can be pepper noise. One of the issues that we incur is distortion by the dilation operator therefore we perform erosion to reduce this negative effect. We can easily visualize the effects of closing.

\subsection{Hole Filling}

Regions detected after segmentation have holes present in them which are present due to the noises. To overcome this issue we opt for closing the image. The segmented image is in binary image form hence the road pixels are labelled as 1 and non-road pixels are labelled as 0 . For filing the small hole we invert the image. After this we search all the areas of the non-road image to compute them using recursive scanning. After deleting the small regions we invert the image. Some regions identified while performing the previous steps still have holes in them and to deal with them a simple algorithm is used which is mentioned above.

\subsection{Small Region Filtering}

Small regions are the areas that do not belong to any part of the road and are distributed all over the image. These regions act as obstruction and hence re not needed because the focus is on road extraction. We can remove these regions by following a simple filtering technique which works on the basis of areas. Regions having area less than a given threshold is eliminated and is not included $\mathrm{n}$ any further processing.

\subsection{Morphological Opening}

The basic effect which opening has on the image is similar to erosion. In this we tend to remove the bright foreground pixels from the regions or edges. However, in comparison to erosion dilation is less destructive. We can perform the same exact operations done by morphological operators by using structuring elements. The effect can be stated as elimination of all the other regions whose shape does not matches to our structuring element or the regions which are not able to contain our structuring element. Opening can be performed by erosion followed by dilation and using the same structuring element for both the operations. Therefore, similar to the erosion operator the opening operator requires two inputs one being input image and the other being structuring element. Its the dual of closing that means opening the bright foreground pixels with the structuring element is equivalent to closing the dark background pixels with the sameelement.

\subsection{Length based Region Filtering}

There are two kinds of unwanted linear structures to remove from the road to improve its representation. In this step linear structure that have lengths less than a given minimum threshold is eliminated. Also, linear structures which are long and isolated are removed and are not processed any further.

\subsection{Branch Removal}

There are many small non-road and linear structures which are attached with the main structure of the road. These can be differentiated from the road because they are connected on one end with the road and their other end is free. The length of the structures is also small in comparison to the length of the roads. Therefore to increase the accuracy of the detected output we remove these structures. 


\subsection{Segment Linking}

In this process two segments which have to be merged are identified and then we try and locate all the pixels that have to be mapped into the road pixels. The discontinuities are also identified to help us connect them to the road pixels on the basis of orientation and distances. Once the segment which have to be mapped is identified the pixels are located and mapped into the already existing road pixels.

\subsection{Morphological Thinning}

It is a process which is done usually only on an binary image. As the name suggests the process is done to regularize the output which we have got from the previous step. By regularizing we mean that it clears up the edges of the detected road to make the output seem more in line with the actual road pixels of the original image. Just like other morphological operators thinning is also performed using structuring element that means it also requires 2 inputs one, image and two, structuring element. This helps in eroding the boundary of the foreground structures as effectively as possible.

\section{Experimental Results and Discussions}

\subsection{Performance Parameters}

\subsubsection{Description}

The performance evaluation of the target image with the output image is done using the peak signal to noise ratio, Normalized cross co-relation, structural content, complete- ness, correctness and accuracy. All the given parameters are used to plot graphs and compare the efficiency of 5 different algorithms. The parameters are descried one by one below.

\subsection{Peak Signal to Noise Ratio}

Peak signal to noise ratio is also called as PSNR and is used for measuring peak error between the input image and the output image. It is calculated by finding the ratio be- tween maximum possible power of the signal and power of the noise because of which the image gets corrupted. The higher the value of PSNR the effective the algorithm works hence higher value of PSNR indicated that the image which is formed possess higher quality. There are also some exceptions like in edge detect ions where it should be less.

The Formula for MSE is:

$\sum_{i=M, N}\left[\frac{[I 1(m, n)-I 2(m, n)]^{2}}{M \times N}\right]$

It can be calculated by using the formula PSNR $=10^{*} \log 10(\mathrm{R} 2$ /MSE). Here $\mathrm{R}$ indicated the Maximum variation possible in the input image. So $\mathrm{R}$ is 255 if the data type is of 8 bit and unsigned integer.

\subsection{Normalized Cross Correlation}

Normalized Cross Correlation can also be termed as NCC. It can be defined as measure of similarity between two images by finding out displacement of one with respect to another. This is also known as a sliding dot product or sliding inner-product. Cross correlation can be defined as comparing the matrix for 2 different images about if they are having same maximum or minimum intensities. Normalized cross correlation on the other hand can compare metrics with different range of values. normalized cross correlation has many applications in the field of computer vision which can include motor tracking, stereo vision etc. Normalized correlation is sensitive towards rotation or change in scale. The formula for NCC is:

$\frac{1}{n} \quad \sum_{x, y} \frac{1}{\sigma_{x,} \sigma_{y}}\left(f(x, y)-f^{j}\right)\left(t(x, y)-t^{j}\right)$

\subsection{Structural Content}

Structural Content is used for measuring structural similarity between two images. We can say that its index is a full reference matrix which is defined as the measure of similarity is calculated by using distortion free image. The main purpose of this is to improve the quality of methods such as PSNR and MSE. Techniques like PSNR and MSE calculate absolute error while Structural similarity index is a model based on perception hence it considers image degradation. Structural similarity index outperforms MSE and PSNR in both quality and accuracy. There are various variants of Structural similarity index which are: Multi-Scale, ThreeComponent Structural Dissimilarity and Video Quality metrics. Multi Scale method is a more advanced version of Structural similarity index and hence gives better quality. Three-Component Structural Similarity the other hand is based on human vision and Structural dissimilarity and vedio metrics are its other advanced forms.

\subsection{Completeness}

Completeness is defined as the ratio of total number of true positive pixels with that of summation of all the true positive pixels and false negative pixels. It can also be defined as a ratio of matched reference data to the original data.

$\frac{T P}{(T P+F N)}=\frac{(\text { length of matched reference })}{(\text { length of total reference })}$

True positive(TP):indicates the pixels which are correctly detected by the algorithm that is the pixels which are matched with the road pixels and are road pixels in reality in the original image. False negative(FN): indicates the pixels which should be detected as road pixels but are not detected as such. Another term used in place of completeness is the sensitivity of the image. It means that the pixels are detected as road pixels but are not truly road pixels and are detected falsely as one.

\subsection{Correctness}

Correctness can be defined as the ratio of no of true positive pixels to that of the sum of the true positive pixels and false positive pixels. It can also be defined as ratio of correctly extracted road to extracted road to give the percentage of correctness in the detected output.

$\frac{T P}{(T P+F P)}=\frac{(\text { length of matched extraction })}{(\text { length of extraction })}$

False positive(FP): indicates the pixels which are not actually part of the road but are detected as road. Another term used in place of correctness is specificity. It means that the algorithm detects certain pixel as road pixel but in reality they are false indications because they do not belong to road pixels. True positive(TP):indicates the pixels which are correctly detected by the algorithm that is the pixels which are matched with the road pixels and are road pixels in reality in the original image.

\subsection{Accuracy}

Accuracy of the image is defined by the ratio of sum of true positive and true negative pixels to that of sum of true positive, true negative, false positive and false negative pixels. 
$\frac{(T P+T N)}{(T P+F P+T N+F N)}=\frac{(\text { length of matched extraction })}{\text { (length of extracted data }+ \text { length of unmatched reference) }}$

True negatives (TN): are described as those pixels which are a part of road but are not detected as road pixels. It means that the pixels which are described as non road pixels and are correctly detected so because in original image also they are non- road pixels are true negative pixels. True positive(TP):indicates the pixels which are correctly detected by the algorithm that is the pixels which are matched with the road pixels and are road pixels in reality in the original image.

\section{Datasets}

\subsection{Description}

The data sets displayed below contain information gathered from the statistical analysis part of the project. All the images from our data set are tested on the basis of the following parameters- completeness, correctness, accuracy, peak signal to noise ratio, structural content and normalized cross co-relation. Here, we are presenting the values obtained after applying the algorithms for the above mentioned parameters for each and every image in our data set. This data set has provided us with enough information to analyze the pros and cons of each algorithm and to represent it graphically to decide how efficient and accurate the algorithms are as compared to each other. In the table 5.1 we have given data regarding correctness of each and every image present in our dataset. In this table there are 5 columns each for Sobel (S), Canny(C), Connected Component Analysis (CCA), K-Means Segmentation and Roberts edge detection respectively. It also has 17 rows each representing 1 image from our dataset and values for the following 6 parameters are mentioned in the respective row. Similarly for all the other tables which are 5.2 for Completeness 5.3 for Accuracy 5.4 for Normalized Cross Correlation 5.5 for Structural Content and 5.6 For Peak Signal To Noise Ratio(PSNR)same criteria has been followed. A complete table for our dataset which has helped us in analyzing and comparing above mentioned algorithm and providing a valid conclusion has been presented.

Table 5.1: Correctness Data

\begin{tabular}{|c|c|c|c|c|c|}
\hline \multicolumn{7}{|c|}{$\begin{array}{c}\text { Correctness } \\
\text { No. }\end{array}$} & $\mathbf{S}$ & $\mathbf{C}$ & $\mathbf{C C A}$ & K Means & Roberts \\
\hline 1 & 59.269 & 60.359 & 67.464 & 29.068 & 62.83 \\
\hline 2 & 49.353 & 51.387 & 71.508 & 37.434 & 49.239 \\
\hline 3 & 48.385 & 48.389 & 88.135 & 72.311 & 59.928 \\
\hline 4 & 62.797 & 62.152 & 57.1338 & 45.008 & 38.242 \\
\hline 5 & 30.87 & 30.274 & 48.665 & 36.881 & 30.992 \\
\hline 6 & 20.276 & 20.316 & 69.59 & 29.502 & 38.473 \\
\hline 7 & 17.115 & 17.142 & 42.861 & 27.609 & 23.953 \\
\hline 8 & 26.254 & 26.873 & 35.027 & 47.773 & 27.654 \\
\hline 9 & 21.888 & 22.203 & 34.735 & 20.956 & 23.953 \\
\hline 10 & 20.193 & 20.305 & 33.067 & 20.716 & 21.03 \\
\hline 11 & 35.879 & 35.948 & 73.271 & 49.958 & 35.74 \\
\hline 12 & 65.243 & 63.98 & 70.621 & 54.779 & 63.596 \\
\hline 13 & 38.532 & 39.643 & 67.685 & 25.028 & 39.53 \\
\hline 14 & 36.266 & 37.156 & 71.744 & 40.468 & 36.753 \\
\hline 15 & 36.959 & 37.943 & 67.45 & 59.521 & 36.841 \\
\hline 16 & 25.577 & 25.678 & 44.579 & 41.089 & 25.609 \\
\hline 17 & 39.125 & 40.601 & 64.467 & 51.568 & 39.544 \\
\hline
\end{tabular}

Table 5.2: Completeness Data

\begin{tabular}{|c|c|c|c|c|c|}
\hline & & & Completeness & & \\
\hline $\begin{array}{c}\text { Image } \\
\text { No. }\end{array}$ & $\mathbf{S}$ & $\mathbf{C}$ & $\mathbf{C C A}$ & K Means & Roberts \\
\hline 1 & 44.504 & 45.543 & 66.125 & 81.58 & 48.635 \\
\hline 2 & 45.903 & 51.577 & 81.497 & 75.361 & 47.671 \\
\hline 3 & 53.648 & 58.416 & 80.417 & 80.471 & 45.651 \\
\hline 4 & 48.49 & 48.342 & 52.093 & 82.335 & 18.294 \\
\hline
\end{tabular}

\begin{tabular}{|c|c|c|c|c|c|}
\hline 5 & 45.282 & 44.877 & 64.327 & 67.363 & 41.858 \\
\hline 6 & 37.271 & 39.379 & 73.955 & 85.1 & 56.467 \\
\hline 7 & 36.545 & 36.663 & 81.339 & 76.672 & 44.346 \\
\hline 8 & 47.964 & 53.945 & 67.502 & 77.583 & 52.037 \\
\hline 9 & 30.512 & 31.003 & 44.329 & 43.586 & 33.924 \\
\hline 10 & 35.001 & 36.333 & 40.418 & 50.994 & 32.357 \\
\hline 11 & 29.435 & 31.749 & 74.689 & 85.244 & 32.173 \\
\hline 12 & 48.272 & 50.688 & 51.333 & 75.292 & 52.711 \\
\hline 13 & 48.625 & 52.765 & 80.821 & 76.147 & 52.742 \\
\hline 14 & 33.73 & 36.732 & 45.665 & 75.638 & 37.541 \\
\hline 15 & 37.496 & 41.331 & 89.602 & 88.132 & 40.258 \\
\hline 16 & 50.401 & 53.2 & 72.137 & 71.65 & 50.936 \\
\hline 17 & 51.753 & 57.641 & 86.257 & 85.674 & 56.147 \\
\hline
\end{tabular}

Table 5.3: Accuracy Data

\begin{tabular}{|c|c|c|c|c|c|}
\hline \multicolumn{6}{|c|}{ Accuracy } \\
\hline \begin{tabular}{|c|} 
Image \\
No.
\end{tabular} & $\mathbf{S}$ & $\mathrm{C}$ & CCA & K Means & Roberts \\
\hline \begin{tabular}{|l|}
1 \\
\end{tabular} & 80.598 & 80.984 & 86.665 & 55.899 & 83.751 \\
\hline 2 & 77.42 & 78.31 & 88.626 & 66.4 & 77.359 \\
\hline 3 & 66.013 & 65.913 & 93.146 & 88.653 & 80.869 \\
\hline 4 & 83.73 & 83.556 & 71.459 & 61.196 & 63.498 \\
\hline 5 & 63.198 & 62.641 & 75.595 & 65.13 & 64.324 \\
\hline 6 & 57.327 & 56.11 & 88.09 & 55.461 & 72.689 \\
\hline 7 & 64.783 & 64.767 & 81.385 & 67.138 & 63.806 \\
\hline 8 & 51.387 & 49.802 & 58.95 & 72.088 & 52.08 \\
\hline 9 & 62.849 & 63.005 & 71.058 & 54.01 & 63.806 \\
\hline 10 & 61.986 & 61.426 & 73.565 & 54.351 & 64.637 \\
\hline 11 & 56.763 & 56.183 & 81.55 & 64.846 & 55.884 \\
\hline 12 & 81.19 & 81.091 & 82.993 & 78.902 & 81.185 \\
\hline 13 & 76.574 & 76.823 & 89.505 & 54.225 & 76.756 \\
\hline 14 & 75.751 & 75.78 & 86.032 & 73.803 & 75.459 \\
\hline 15 & 69.384 & 69.431 & 87.014 & 82.616 & 68.827 \\
\hline 16 & 58.04 & 57.071 & 74.868 & 71.975 & 57.874 \\
\hline 17 & 74.873 & 75.279 & 88.041 & 81.503 & 74.693 \\
\hline
\end{tabular}

Table 5.4: Normalized Cross Correlation Data

\begin{tabular}{|c|c|c|c|c|c|}
\hline \multicolumn{6}{|c|}{$\mathrm{NCC}$} \\
\hline $\begin{array}{c}\text { Image } \\
\text { No. }\end{array}$ & $\mathbf{S}$ & $\mathrm{C}$ & CCA & K Means & Roberts \\
\hline \begin{tabular}{l|}
1 \\
\end{tabular} & 0.23744 & 0.24672 & 0.6758 & 0.85139 & 0.24909 \\
\hline 2 & 0.20677 & 0.2833 & 0.80631 & 0.76965 & 0.21753 \\
\hline 3 & 0.18805 & 0.19072 & 0.82269 & 0.82957 & 0.19472 \\
\hline 4 & 0.2922 & 0.35121 & 0.4853 & 0.84303 & 0.074693 \\
\hline 5 & 0.20031 & 0,20853 & 0.58716 & 0.64434 & 0.16035 \\
\hline 6 & 0.12765 & 0.20396 & 0.73062 & 0.8419 & 0.28665 \\
\hline 7 & 0.18734 & 0.19767 & 0.85426 & 0.77232 & 0.10789 \\
\hline 8 & 0.18592 & 0.2804 & 0.59285 & 0.77326 & 0.24285 \\
\hline 9 & 0.16473 & 0.16864 & 0.3829 & 0.36048 & 0.12873 \\
\hline 10 & 0.18294 & 0.20152 & 0.34257 & 0.40387 & 0.13201 \\
\hline 11 & 0.067057 & 0.08848 & 0.77305 & 0.90217 & 0.1 \\
\hline 12 & 0.20892 & 0.25361 & 0.51181 & 0.81945 & 0.28065 \\
\hline 13 & 0.19637 & 0.25468 & 0.79762 & 0.68155 & 0.2503 \\
\hline 14 & 0.10808 & 0.13756 & 0.4367 & 0.75736 & 0.1524 \\
\hline 15 & 0.11477 & 0.15037 & 0.92893 & 0.92116 & 0.14103 \\
\hline 16 & 0.19346 & 0.23014 & 0.67613 & 0.71366 & 0.19249 \\
\hline 17 & 0.21443 & 0.29137 & 0.88282 & 0.8855 & 0.26373 \\
\hline
\end{tabular}

Table 5.5: Structural Content Data

\begin{tabular}{|c|c|c|c|c|c|}
\hline $\begin{array}{c}|c| \\
\text { Image } \\
\text { No. }\end{array}$ & $\mathbf{S}$ & $\mathbf{C}$ & $\mathbf{C C A}$ & K Means & Roberts \\
\hline 1 & 2.6905 & 2.5052 & 1.0241 & 0.30225 & 2.5163 \\
\hline 2 & 2.0047 & 1.616 & 0.91694 & 0.50987 & 1.8281 \\
\hline 3 & 2.3686 & 2.3665 & 1.125 & 0.93074 & 2.272 \\
\hline 4 & 1.6538 & 1.2997 & 1.211 & 0.51275 & 4.5138 \\
\hline 5 & 1.2765 & 1.2119 & 0.8406 & 0.57369 & 1.6512 \\
\hline 6 & 1.0529 & 0.84534 & 1.0066 & 0.32464 & 1.0551 \\
\hline 7 & 0.64498 & 0.62435 & 0.5058 & 0.31047 & 0.68928 \\
\hline 8 & 1.0228 & 0.74592 & 0.52047 & 0.59903 & 0.95759 \\
\hline 9 & 1.1876 & 1.1695 & 0.79447 & 0.50049 & 1.4699 \\
\hline 10 & 1.0158 & 0.94315 & 0.92773 & 0.44036 & 1.2463 \\
\hline 11 & 2.479 & 1.9817 & 0.93794 & 0.52971 & 1.8173 \\
\hline 12 & 2.9233 & 2.3356 & 1.3965 & 0.62673 & 2.0251 \\
\hline 13 & 1.316 & 1.1433 & 0.87097 & 0.33469 & 1.1358 \\
\hline
\end{tabular}




\begin{tabular}{|l|c|c|c|c|c|}
\hline 14 & 2.3549 & 1.9264 & 1.6839 & 0.50255 & 1.7261 \\
\hline 15 & 1.9762 & 1.6232 & 0.71106 & 0.60861 & 1.5606 \\
\hline 16 & 0.91827 & 0.76803 & 0.61519 & 0.51783 & 0.9056 \\
\hline 17 & 1.3068 & 1.0453 & 0.74833 & 0.58695 & 1.0473 \\
\hline
\end{tabular}

Table 5.6: Peak Signal to Noise Ratio Data

\begin{tabular}{|c|c|c|c|c|c|}
\hline & & & PSNR & & \\
\hline & S & C & CCA & K Means & Roberts \\
\hline 1 & 8.6253 & 8.5822 & 10.194 & 3.9929 & 8.6135 \\
\hline 2 & 6.9201 & 7.0544 & 10.4815 & 5.7466 & 6.8147 \\
\hline 3 & 7.062 & 7.0826 & 13.3929 & 11.0743 & 7.0429 \\
\hline 4 & 5.5271 & 5.3327 & 6.2939 & 4.5962 & 5.3117 \\
\hline 5 & 5.4714 & 5.3925 & 6.6128 & 5.52519 & 5.79 \\
\hline 6 & 5.8229 & 5.4357 & 10.667 & 4.1318 & 6.5465 \\
\hline 7 & 6.5458 & 6.4853 & 8.8888 & 5.6466 & 6.4291 \\
\hline 8 & 4.9431 & 4.4964 & 4.6055 & 6.497 & 5.0728 \\
\hline 9 & 6.0356 & 6.0206 & 6.0924 & 4.2589 & 6.301 \\
\hline 10 & 5.9943 & 5.8917 & 6.6468 & 4 & 6 \\
\hline 11 & 4.6579 & 4.4626 & 8.5328 & 5.3453 & 4.3893 \\
\hline 12 & 8.0057 & 8.0212 & 9.2597 & 7.8558 & 7.9669 \\
\hline 13 & 6.9554 & 6.9613 & 10.887 & 4.1226 & 6.9151 \\
\hline 14 & 7.2937 & 7.168 & 9.54 & 6.4278 & 7.0627 \\
\hline 15 & 6.1055 & 5.9753 & 9.774 & 8.1304 & 5.8342 \\
\hline 16 & 5.6656 & 5.3231 & 6.9263 & 6.2035 & 5.622 \\
\hline 17 & 6.7934 & 6.673 & 10.4888 & 8.3552 & 6.5073 \\
\hline
\end{tabular}

\section{Inputs and Outputs}

\subsection{Connected Component Analysis}
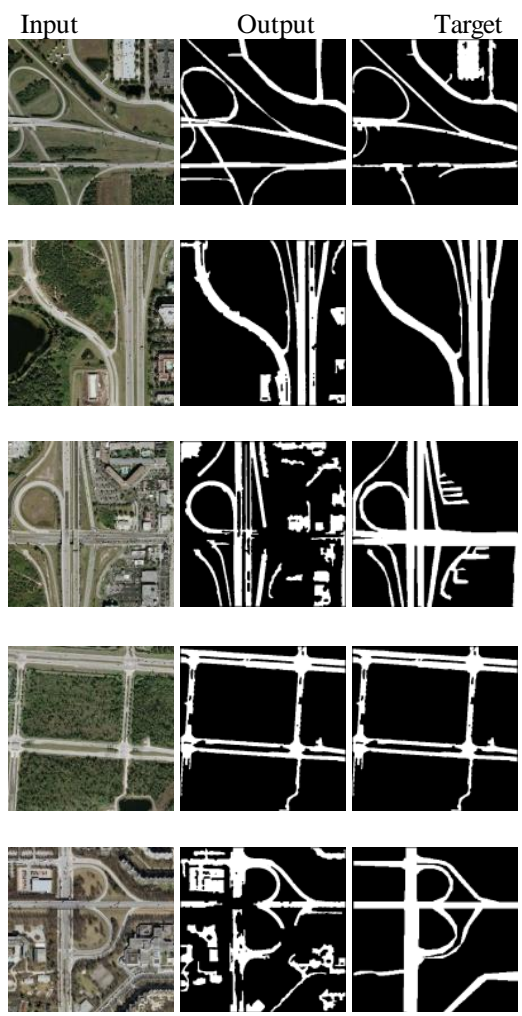

Fig. 6.1: Input, Output and Target images of Developing ub-Urban DataSet
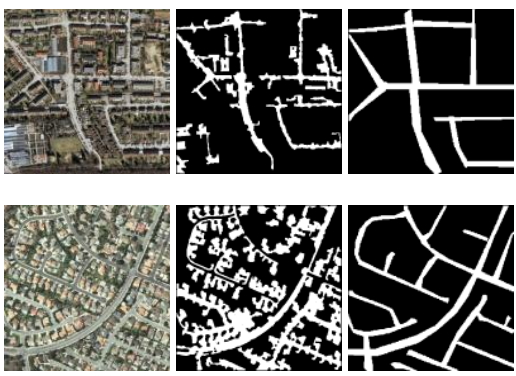
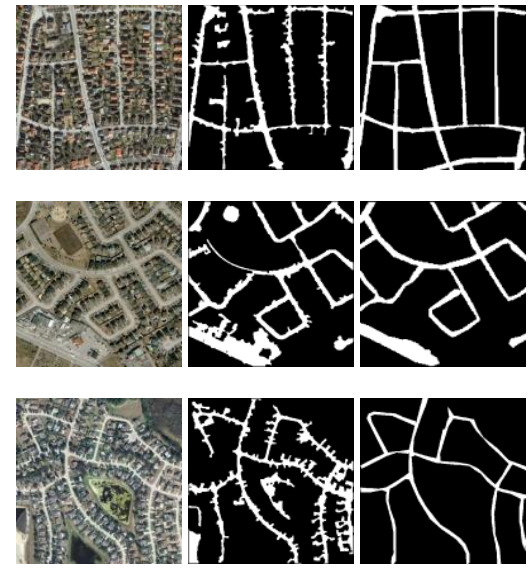

Fig. 6.2: Input, Output and Target images of Developing Urban Data-Set
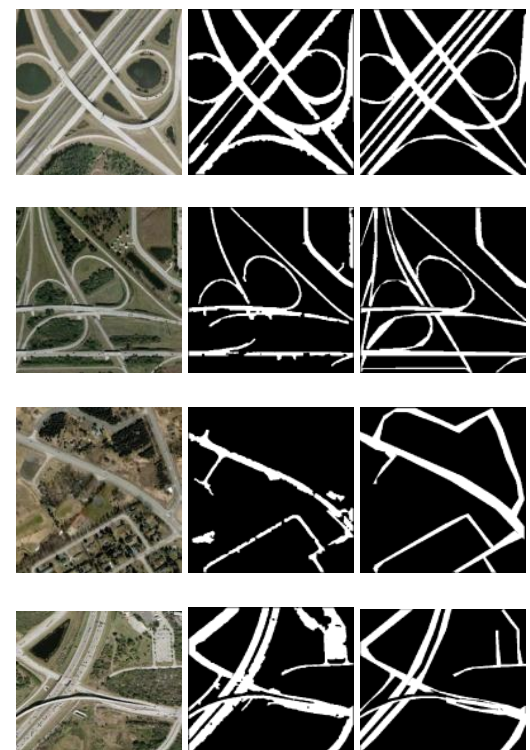

Fig. 6.3: Input, Output and Target images of Developed Sub-Urban DataSet
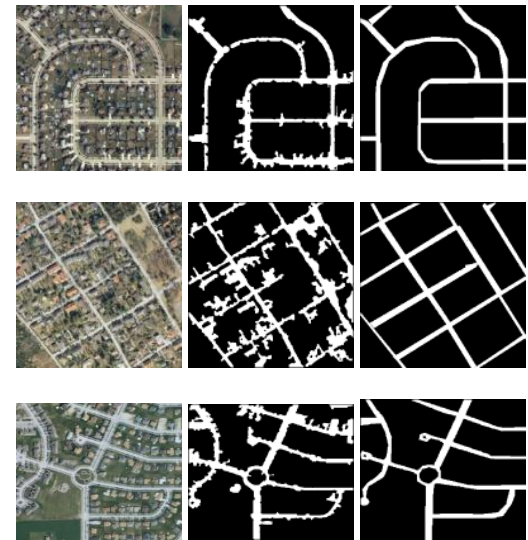

Fig. 6.4: Input, Output and Target images of Developed Urban Data-Set

\section{Statistical Analysis}

In this section we are representing the data gathered from the images above in terms of completeness, correctness, accuracy, normalized cross correlation ratio, structural con- tent and peak signal to noise ratio graphically.

\subsection{Specific comparison}

In the first section the comparison between the different road detection techniques is described specifically so as to see which 
technique showed the better performance parameter wise. The plots represented below are directly taken from the output of the statistical analysis test graphical user interface. In this comparison it is difficult to interpret which method is the best among all the other methods overall but it still gives a very fair idea of how the methods compare against each other on specific image level. From the below given figures it is more evident that the methods $\mathrm{K}$ Means and connected component analysis are more neck to neck with each other in all comparisons and in all images. To further clarify so as to which method is dominant over the other or suits our data set more conveniently we can compare the average stats taken from our data and compare the methods easily on a more personal basis as shown in Fig. 7.1 to Fig. 7.6. In Fig 7.1-7.6 the xaxis denotes the image number and y axis denotes the numerical value of the data obtained for particular image.

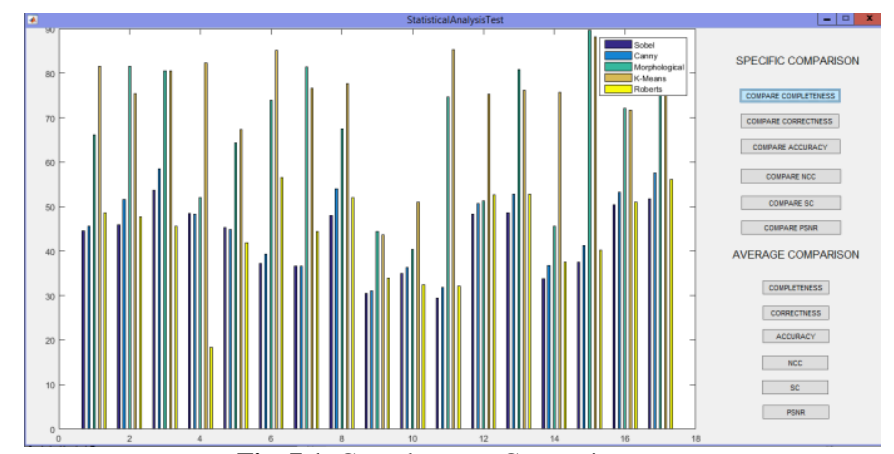

Fig. 7.1: Completeness Comparison

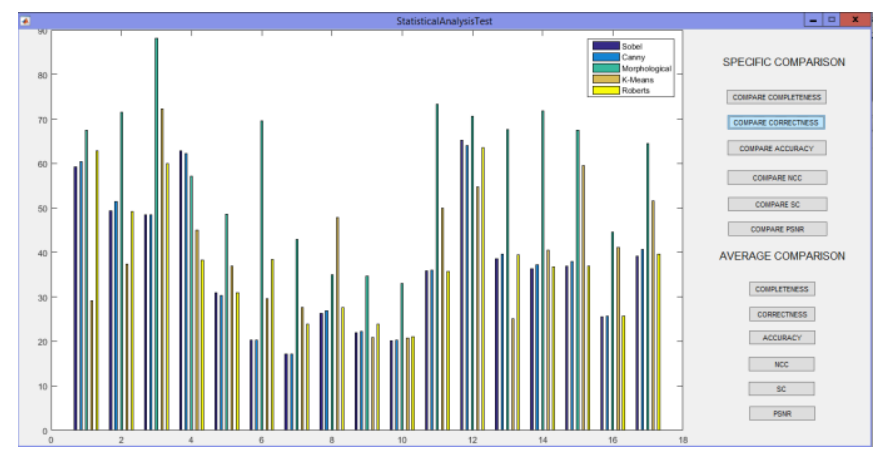

Fig. 7.2: Correctness comparison

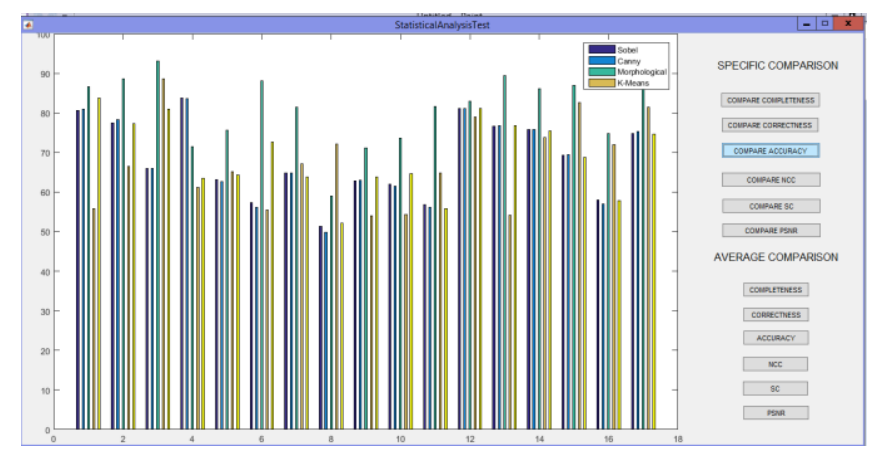

Fig. 7.3: Accuracy Comparison

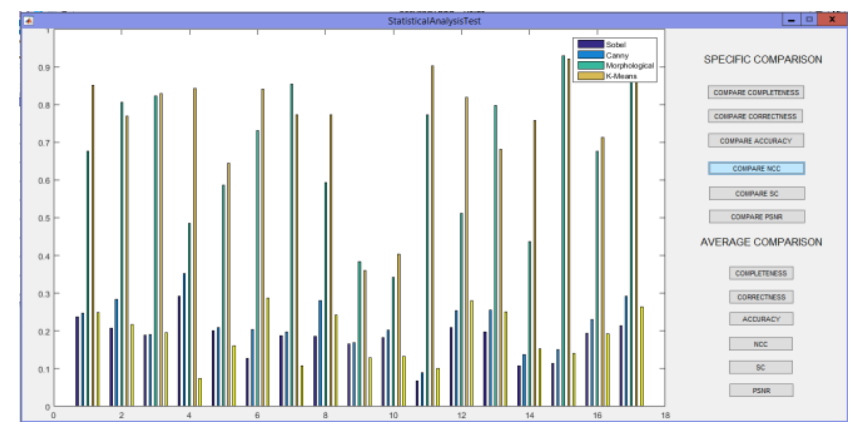

Fig. 7.4: NCC Comparison

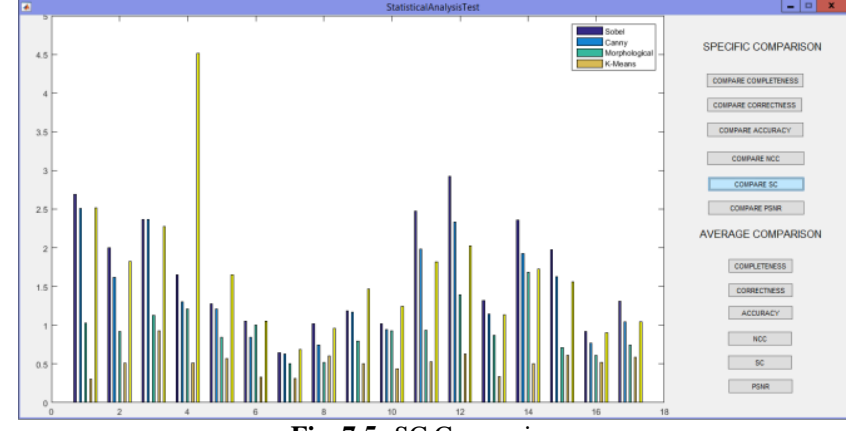

Fig. 7.5: SC Comparison

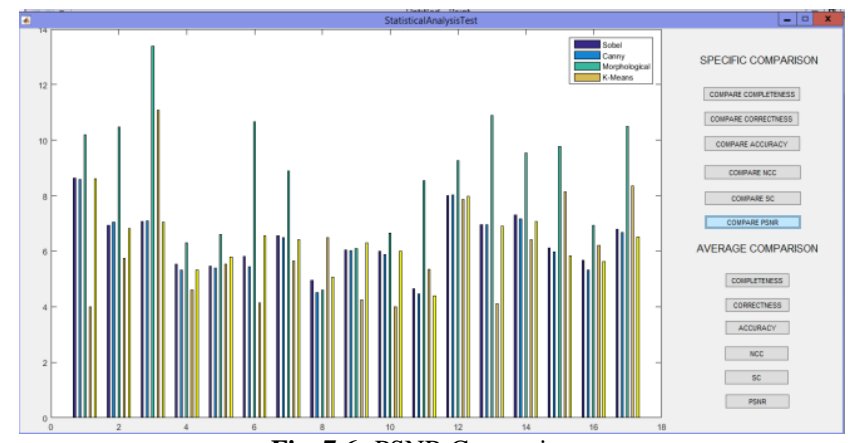

Fig. 7.6: PSNR Comparison

\subsection{Average comparison}

From the above section and figures pertaining to the above section we have clearly established that the K-Means method and the connected component analysis method are more closer to each other in terms of accuracy and efficiency. This graphical representation simplified our analysis by providing us with clear outlook about the proper performance parameters of each and every method which helped us reach our conclusion more faster than usual. (from Fig.7.7- 7.12). In fig8.7-8.12 the x-axis denotes the methods used for road detection where in 1-Sobel, 2- Canny, 3-Connected Component Analysis, 4-K-Means and 5-Roberts.

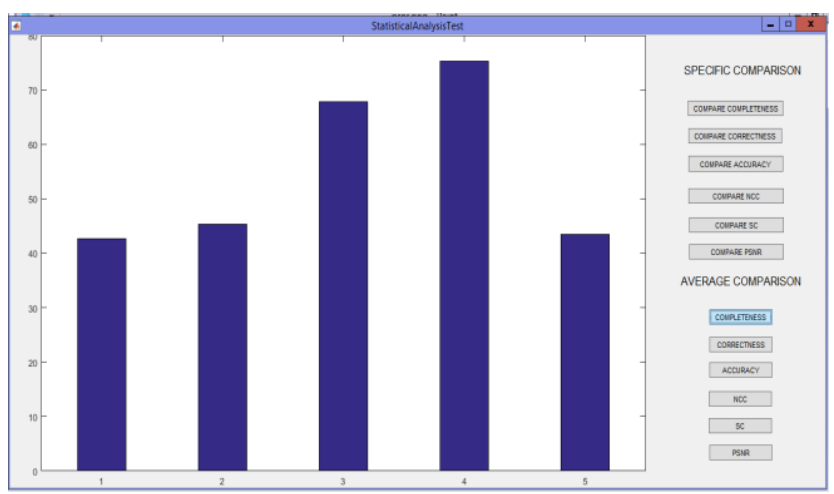

Fig. 7.7: Average Completeness

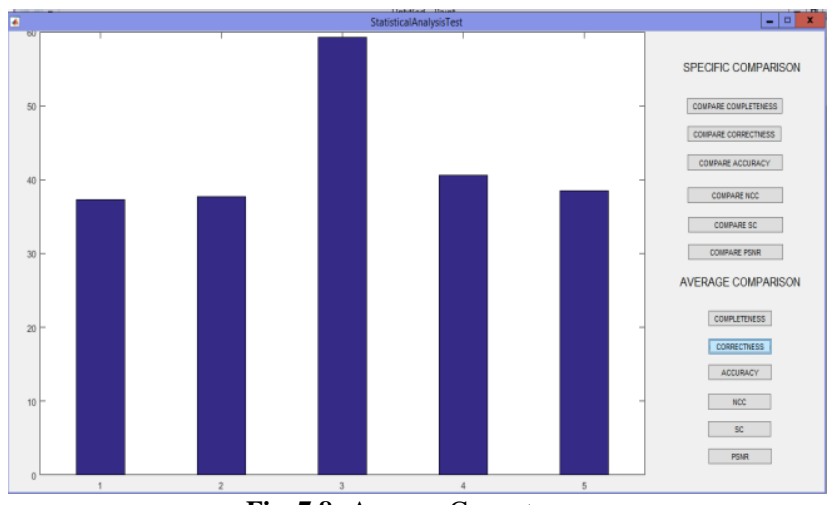

Fig. 7.8: Average Correctness 


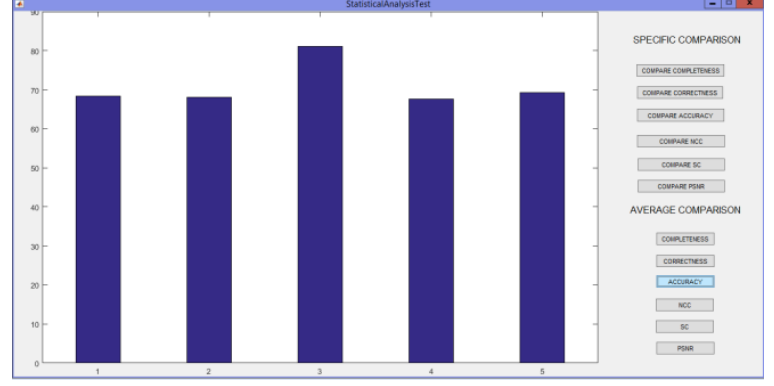

Fig. 7.9: Average Accuracy

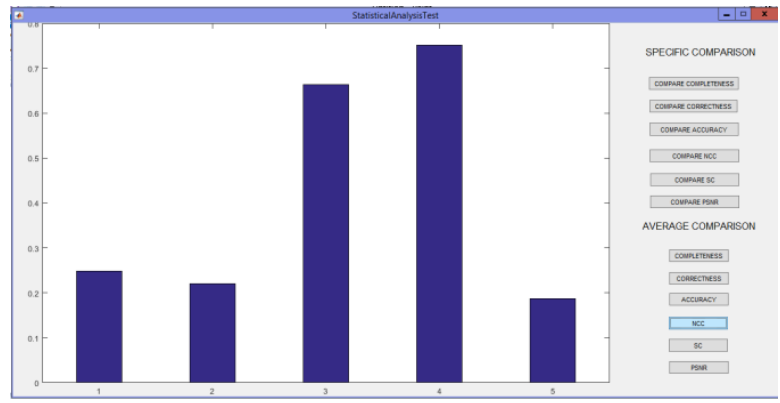

Fig. 7.10: Average NCC

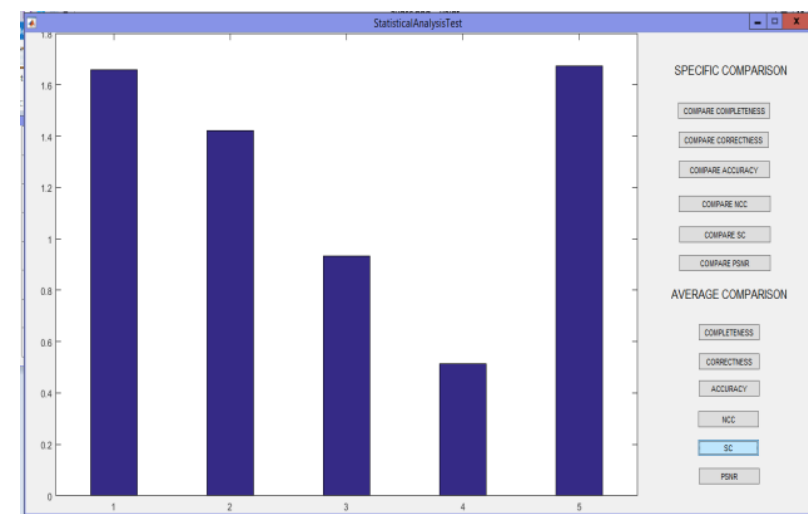

Fig. 7.11: Average SC

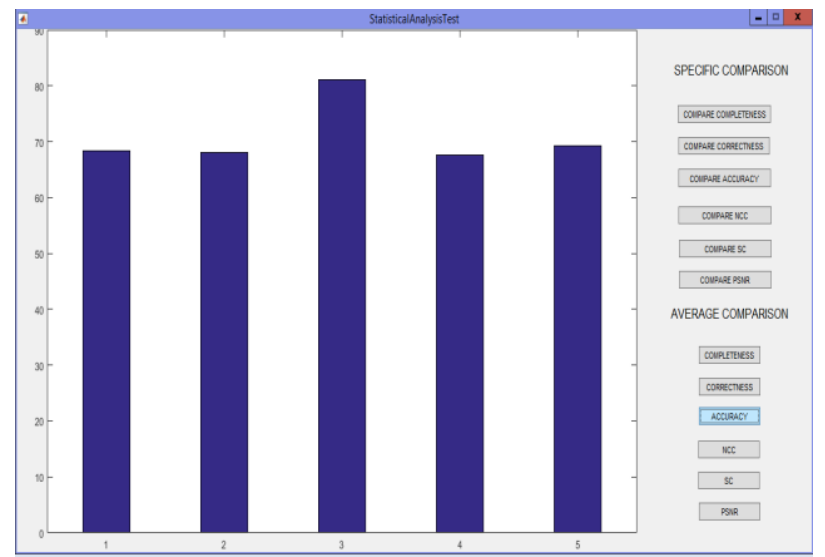

Fig. 7.12: Average PSNR

\section{Conclusion and Future Enhancement}

The prime conclusion which can be drawn from the research work is that connected component analysis method is more effective and efficient method of extracting roads from the dataset as the outputs from the images are the closest to the target output. The method implemented outshines the other road detection algorithms namely K-Means, Sobel, Canny and Roberts. The average accuracy of road detection obtained from the connected component analysis method is 81 percent. This proposed method of detecting roads works best with images containing developing suburban and developing urban sectors of the road.

In the scope of future enhancement it can be mentioned that the project can be made more effective by optimizing the output of the algorithm by using evolutionary algorithms such as Particle Swarm optimization, Cuckoo method etc. The adaptive global thresholding technique implemented can be made more rigorous and constricted which can help increase the overall accuracy of the output manifold. Finally, the last enhancement which can be made to make the project more usable is by connecting the project with a real time satellite feed which provides high resolution satellite images at the go and can help clarify the output of the algorithm in a more effective and enhanced way.

\section{References}

[1] Zelang Miao, Bin Wang, Wenzhong Shi, Hua Zhang, A SemiAutomatic Method for Road Centreline Extraction From VHR Images, IEEE Geoscience and Remote Sensing Letters, Vol.11, Issue.11, 2014

[2] Volodymyr Mnih, Geoffrey E. Hinton, Learning to Detect Roads in High-Resolution Aerial Images , Springer Computer Vision âA S ECCV 2010, Lecture Notes in Computer Science, vol.6316, 2010

[3] Amir Robati, Gholam Abbas Barani, Hossein Nzam Abadi Pour, Mohammad Javad Fadaee, Javad Rahimi Pour Anaraki, Balanced fuzzy particle swarm optimization, ScienceDirect Applied Mathematical Modelling, Vol.36, Issue.5, 2012.

[4] P Jaganathan, S Jaiganesh, A Particle Swarm Optimization based fuzzy c means approach for efficient web document clustering, International Journal of Engi- neering and Technology (IJET), Vol.5, 2013.

[5] Minhas, Rashid, et al. A robust object detection approach using boosted anisotropic multi resolution analysis , IEEE Circuits and Systems (MWSCAS), 2011

[6] Qiu, Yufang, Dongping Ming, and Xian Zhang, Object oriented land cover clas- sification combining scale parameter preestimation and mean-shift segmentation, IEEE Geoscience and Remote Sensing Symposium (IGARSS), 2016

[7] Reddy, Chandan K., Jin-Hyeong Park. Multi-resolution boosting for classifica- tion and regression problems. , Knowledge and information systems, 2012

[8] Al-Stouhi, Samir, Chandan K. Reddy. Adaptive boosting for transfer learning us- ing dynamic updates. Springer Joint European Conference on Machine Learning and Knowledge Discovery in Databases, 2011.

[9] Baykal, Nazife, Yasemin YardÄśmcÄśs ÃG etin, Road Extraction From High Resolution Satellite Images Using Adaptive Boosting With Multi-Resolution Analysis, Diss. Middle East Technical University, 2012.

[10] Rizvi, I. Ali, B. Krishna Mohan, Object-oriented method for automatic extraction of road from High Resolution Satellite Images. Iranian Journal of Earth Sciences 2.1, 2010.

[11] Wang, Yanping, et al. An object-oriented method for road damage detection from high resolution remote sensing images, IEEE Geoinformatics, 2011 19th Inter- national Conference on. IEEE, 2011.

[12] Mhangara, Paidamwoyo, John Odindi, Linda Kleyn, and Hardly Remas. Road extraction using object oriented classification , 2011.

[13] Hao, Ying, Li-qiang Wang, Xi'an Zhao, Automatic roads extraction from high- resolution remote sensing images based on SOM, IEEE In Natural Computation (ICNC), 2010 Sixth International Conference on, vol. 3, 2010

[14] Chaudhuri, D., N. K. Kushwaha, A. Samal, Semi-automated road detection from high resolution satellite images by directional morphological enhancement and segmentation techniques, IEEE journal of selected topics in applied earth obser- vations and remote sensing 5, no. 5, 2012

[15] Valero, Silvia, Jocelyn Chanussot, Jon Atli Benediktsson, Hugues Talbot, BjÃürn Waske, Advanced directional mathematical morphology for the detection of the road network in very high resolution remote sensing images, Pattern Recognition Letters 31, no. 10, 2010

[16] Liu, Bo, Huayi Wu, Yandong Wang, Wenming Liu, Main road extraction from zy-3 grayscale imagery based on directional mathematical morphology and vgi prior knowledge in urban areas, PloS one 10 , no. 9,2015 
[17] Negri, Matteo, Paolo Gamba, Gianni Lisini, Florence Tupin, Junction-aware extraction and regularization of urban road networks in high-resolution SAR images, IEEE Transactions on Geoscience and Remote Sensing 44, no. 10, 2006.

[18] Asef, Mostafa Okauti, Automatic road extraction based on neurofuzzy algorithm, In Proceedings of the 10th WSEAS Int. Conference on Robotics, Control and Manufacturing Technology, 2010.

[19] Gao Wenshuo, Zhang Xiaoguang, Yang Lei, Liu Huizhong, "An improved Sobel edge detection", IEEE trans. Computer Science and Information Technology (lCCSIT) 20103 rd IEEE International Conference on, vol. 9, no. 5, pp. 67-71, 2010.

[20] William McIlhagga, "The Canny Edge Detector Revisited, " International Journal of Computer Vision, no.91,pp.251-261,2011

[21] Xiao W, Hui X. An Improved Canny Edge Detection Algorithm Based on Predisposal Method for Image Corrupted by Gaussian Noise. IEEE World Automation Congr. 2010; p. 113-116.

[22] Wang HR, Yang JL, Sun HJ, Chen D, Liu XL. An improved Region Growing Method for Medical Image Selection and Evaluation Based on Canny Edge Detection. IEEE Int. Conf. Manage. and Service Sci.2011; p. 1-4, DOI: 10.1109/ICMSS. 2011.5999180. 